\author{
Marco Carotenuto \\ Vincenzo Guidetti \\ Francesca Ruju \\ Federica Galli \\ Francesca R. Tagliente \\ Antonio Pascotto
}

\section{Headache disorders as risk factors for sleep disturbances in school aged children}

Published online: 20 July 2005

\section{Carotenuto (两)}

Dipartimento di Psichiatria,

Neuropsichiatria infantile, Audiofoniatria, Dermatovenereologia,

Cattedra di Neuropsichiatria Infantile,

Via Pansini 5 PAD XI, I-80131 Naples, Italy

e-mail: marco.carotenuto@unina2.it

Tel.: +39-081-5666692

M. Carotenuto • F. Ruju • A. Pascotto Department of Developmental Neurology and Psychiatry,

Second University of Naples, Naples, Italy

V. Guidetti • F. Galli • F.R. Tagliente

Department of Developmental Neurology and Psychiatry,

University of Rome "La Sapienza",

Rome, Italy

\begin{abstract}
Several epidemiological studies have shown the presence of comorbidity between various types of sleep disorders and different headache subtypes. Migraine without aura is a sensitive risk factor for disorders of initiating and maintaining sleep (odds ratio (OR) 8.2500), and chronic tension-type headache for sleep breathing disorders (OR 15.231), but headache disorder is a cumulative risk factor for disorders of excessive somnolence (OR 15.061). This result has not been reported in the clinical literature.
\end{abstract}

Key words Sleep $\cdot$ Excessive diurnal sleepiness $\cdot$ Headaches $\cdot$ Sleep Disturbances Scale for Children

\section{Introduction}

Several epidemiological studies have shown the presence of comorbidity between various types of sleep disorders and different headache subtypes. Headaches and sleep disturbances are two of the most common and ubiquitous disorders faced in clinical practice. Frequently encountered in the same patient, the two complaints may have a relationship that is often unclear.

Many different sleep disorders, including circadian rhythm disorders, are associated with headache [1].
Inadequate sleep duration or poor sleep quality are common triggering factors for migraine headaches.

There have been several observations of the co-occurrence of sleep disturbances and headaches in children [2, 3]. In fact, sleep disturbances and migraine are part of a cluster of recurrent clinical symptoms that make up what has been called the periodic syndrome of childhood.

It has been proposed that sleep and migraine may be aspects of a common genetic, pathophysiological or biobehavioural substrate, accounting for the comorbidity of these problems in children. However, while a relationship between migraine headaches and sleep disturbances 
has been found in adults, research with paediatric populations remains limited [4].

The serotonergic system, well known as being central in migraine attacks, demonstrates circadian and circannual rhythmicity and is, like other biorhythms, under the pacemaker control of the CNS. Herein, during the onset of a migraine attack, the urinary excretion of 5-hydroxyindoleacetic acid (5-HIAA), which is the main metabolite of serotonin, increases and platelet 5-HT decreases rapidly; pharmacologic depletion can induce a migraine attack, and intravenous 5-HT can abort acute migraine attacks. Systemic serotonin also decreases during REM sleep when the dorsal raphe is silent, which may account in part for the relationship between REM sleep and migraine. In addition to the clinical association between headache and sleep, their relationship also hinges on whether there is a relationship between the hypothalamus (and other regions crucial to the regulation of sleep) and those areas involved in nociception and migraine pathogenesis, suggesting that dysfunction in the regulation of the sleep-regulating system must be capable of generating headaches.

Children with migraine headaches appear to have a range of sleep disturbances. Insufficient sleep, bruxism and co-sleeping are common. Consistent with prior research, children with migraine experienced significantly more frequent sleep disturbances compared to children from a normative community sample [5]. Children with migraine experienced greater sleep disturbances in all domains including longer sleep onset delay, more bedtime resistance, shorter sleep duration, more daytime sleepiness, more night wakings, greater sleep anxiety, more parasomnias and more sleep-disordered breathing.

Although the mechanism by which sleep deprivation or sleep disruption leads to headache is unknown, the association has been recognised for decades. Dull, frontalaching headaches will develop both in normal subjects and in those who suffer from tension-type headaches who are deprived of sleep [6].

In a recent prospective longitudinal study examining the temporal relationships between daily stress, sleep and headache using time-series analysis with lagged correla- tions, Penzien and colleagues found that altered sleep patterns played a key role in precipitating or exacerbating recurrent headache [7].

The aim of this study is to assess the odds ratio (OR) of developing sleep disorders in school-aged children with headache.

\section{Methods}

One hundred and seventy children aged 5-10 years (mean age 8.12 , $\mathrm{SD} \pm 1.44)$ referred between January 2004 and May 2005 to university third level headache centres in Naples and Rome were studied with the Sleep Disturbance Scale for Children questionnaire (SDSC) [8] to assess sleep habits, and were compared with 170 matched children.

The SDSC consists of 26 items and is composed of 6 sleep disorder subscales: disorders in initiating and maintaining sleep (DIMS), sleep breathing disorders (SBD), disorders of arousal (DA), sleep-wake transition disorders (SWTD), disorders of excessive somnolence (DES) and nocturnal hyperhydrosis (SHY). The SDSC subscales are congruent to the International Classification of Sleep Disorders [9].

Children were selected according to the IHS 2004 criteria, and absence of other neurological or psychiatric disorders (epilepsy, behavioural problems, ADHD, neuromuscular diseases), and were evaluated by clinical interview and examination as well by neurophysiological recordings (wake and sleep EEG). Children were excluded from the study if they had known clinical conditions such as Down's syndrome or craniofacial abnormalities. None of the recruited children were being treated with anticonvulsant or psychoactive drugs.

\section{Results}

Global frequency headache subgroups are: migraine with aura (MA) 20\%, migraine without aura (MO) $38.24 \%$, episodic tension-type headache (ETTH) $19.41 \%$ and chronic tension-type headache (CTTH) $22.35 \%$.

Headache children showed higher scores of DIMS, SBD, DA, SWTD and DES categories while lower SHY

Table 1 Mean scores and standard error of items evaluated with the Sleep Disturbance Scale for Children test

\begin{tabular}{lrrrr}
\hline & Headache & Normal & $F$ & \multicolumn{1}{c}{$p$} \\
\hline Sleep latency & $17.20 \pm 4.5$ & $17.08 \pm 4.6$ & 1.06 & 0.66 \\
Nocturnal sleep & $476.47 \pm 96.52$ & $531.52 \pm 81$ & 1.39 & 0.03 \\
DIMS & $11.60 \pm 4.1$ & $10.68 \pm 3.06$ & 1.79 & $<0.0001$ \\
SBD & $5.34 \pm 3.39$ & $4.07 \pm 1.76$ & 3.68 & $<0.0001$ \\
DA & $4.35 \pm 2.11$ & $3.75 \pm 1.04$ & 1.57 & $<0.0001$ \\
SWTD & $10.4 \pm 3.38$ & $8.82 \pm 3.62$ & 1.14 & 0.36 \\
DES & $9.77 \pm 3.11$ & $6.74 \pm 2.25$ & 1.91 & $<0.0001$ \\
SHY & $2.5 \pm 0.93$ & $2.88 \pm 1.6$ & 2.94 & $<0.001$ \\
\hline
\end{tabular}


scores were observed (Table 1). Nocturnal sleep duration (in minutes) is lower in the headache group (headache group 476.47 \pm 96.52 , normal 531.52 $\pm 81, p=0.03$ ).

No differences were observed for Sleep Latency (headache children 17.20 \pm 4.5 , normal 17.08 \pm 4.6 , $p=0.66$ ). To assess the OR for a single headache subgroup, a logistic regression analysis was performed: $\mathrm{MO}$ is a sensible risk factor for DIMS (OR 8.25), and CTTH for SBD (OR 15.231), but headache disorder is a cumulative risk factor for DES (OR 15.061). This result has not been reported in the clinical literature.

\section{Conclusions}

Several studies on headache in children outlined the comorbidity of headaches and sleep disorders and also a relationship between sleep breathing disorders and migraine or tension-type headache. Few studies have focused on the whole sleep-wake cycle and on the circadian aspects of headache to clarify the close relationship between them, even though clinical evidence suggests the importance of investigating sleep habits in paediatric headache centres.

\section{References}

1. Sahota PK, Dexter JD (1990) Sleep and headache syndromes: a clinical review. Headache 30:80-84

2. Lanzi G, Balottin U, Fazzi E, Rosano FB (1983) The periodic syndrome in pediatric migraine sufferers. Cephalalgia 3:218-220

3. Lanzi G, Zambrino CA, Balottin U, Tagliasacchi M, Vercelli P, Termine C (1997) Periodic syndrome and migraine in children and adolescents. Ital J Neurol Sci 18:283-288

4. Paiva T, Martins P, Batista A, Esperanca P, Martins I (1994) Sleep disturbances in chronic headache patients: a comparison with healthy controls. Headache 5:135-141
5. Bruni O, Fabrizi P, Ottaviano $S$, Cortesi F, Giannotti F, Guidetti V (1997) Prevalence of sleep disorders in childhood and adolescence with headache: a case-control study. Cephalalgia 17:492-498

6. Blau JN (1990) Sleep deprivation headache. Cephalalgia 10:157-160

7. Penzien DB, Rains JC, Andrew ME, Galovski TE, Mohammed Y, Mosley TH (2001) Relationships of daily stress, sleep, and headache: a timeseries analysis. Cephalalgia 21:262-263 (Abstract)
8. Bruni O, Ottaviano S, Guidetti V et al (1996) The Sleep Disturbance Scale for Children (SDSC). Construction and validation of an instrument to evaluate sleep disturbances in childhood and adolescence. J Sleep Res 5:251-261

9. - (1990) International Classification of Sleep Disorders. Diagnostic and Coding Manual. American Sleep Disorders Association, Rochester, MN 